\title{
Steroids for intubated croup masking airway haemangioma
}

\author{
K M Kiff, Q Mok, J Dunne, R C Tasker
}

\begin{abstract}
Recently, the beneficial role of steroids for acute laryngotracheobronchitis has been more clearly defined for both intubated and unintubated patients. However, corticosteroids also improve the clinical signs of airway haemangiomata. Two patients are described who illustrate how this can be a source of diagnostic confusion. (Arch Dis Child 1996; 74: 66-67)
\end{abstract}

Keywords: steroids, airway haemangioma, laryngotracheobronchitis, croup.

\section{Case reports \\ CASE ONE}

An 18 week old girl was admitted from casualty. She had been completely well since birth but for three days had suffered worsening stridor, cough, respiratory effort, and poor feeding. A small haemangioma was noted overlying the right parotid gland. A diagnosis of acute laryngotracheobronchitis (LTB) was made and she was started on steroids. A nasopharyngeal aspirate failed to demonstrate any respiratory tract viruses. After further deterioration she was intubated with a $3.0 \mathrm{~mm}$ nasotracheal tube. Laryngoscopy was normal. After five days on the paediatric intensive care unit (PICU) a low pressure leak developed around the endotracheal tube, so she was extubated and the steroids were stopped. At discharge two days later, she had only a croupy cough on crying.

She was readmitted within one week suffering a recurrence of her original symptoms. She again required endotracheal intubation. A microlaryngobronchoscopy revealed a haemangioma originating in the subglottic region and extending to within two centimetres of the carina. Its size and position made it difficult to inject so postoperatively she was started on systemic steroids in an effort to shrink its mass. At further microlaryngobronchoscopy examinations the lesion was injected with methylprednisolone to good effect.

Paediatric Intensive Care Unit, Great Ormond Street Hospital for Children, Great Ormond Street, London WC1N 3JH London W

Q Mok

J Dunne

R C Tasker

Correspondence to: Dr Tasker.

Accepted 27 September 1995

\section{CASE TWO}

A 2 month old baby girl with no previous medical problems presented to casualty. For one week she had been restless, feeding poorly, and had suffered worsening croupy cough and stridor. Skin examination was normal and she was afebrile. She was diagnosed as having acute LTB and treated with dexamethasone and nebulised adrenaline. After admission she deteriorated and required respiratory support: she was intubated with a $2.5 \mathrm{~mm}$ endotracheal tube. After four days of continuous positive airways pressure on the PICU she was extubated. Within two days she was at home with only a mildly croupy cough.

Twelve days later she was readmitted with a marked worsening of her original symptoms. She required emergency intubation, again with a $2.5 \mathrm{~mm}$ endotracheal tube. A microlaryngobronchoscopy revealed a subglottic haemangioma occluding more than $60 \%$ of the lumen. The lesion was injected with methylprednisolone and the child was returned to the PICU for a further seven days of intubation. Subsequent microlaryngobronchoscopy examination showed a marked reduction of the size of the tumour.

\section{Discussion}

Airway haemangiomata and acute LTB share many common physical signs and symptoms, leading to diagnostic confusion.

Acute LTB is a common cause of upper airway obstruction in children. It is unusual before 3 months of age but common until the third year. Often noted in winter months, it may occur in epidemics. LTB usually begins with the signs of an upper respiratory tract infection: fever, a gradual onset of a hoarse cough, stridor, and thin copious secretions. The clinical severity is variable: in the majority the disease is uncomplicated and self limiting but in a few, the airway is seriously compromised. The diagnosis is made more likely by the presence of a funnel shaped narrowing of the glottic and subglottic region on an inspiratory frontal radiograph.

Subglottic haemangiomata although rare, are the most common tumours of the head and neck in small children. They may produce significant mortality and morbidity but tend to regress after 1 year of age. More common in females, more than $50 \%$ of patients display associated cutaneous lesions around the head and neck. Patients usually present with recurrent episodes of croup. The lesions may engorge when the child cries resulting in symptomatic deterioration. There may be feeding problems and generally the clinical severity is related to the size of the tumour. Symptoms may also be exacerbated by concomitant upper airway infection. The diagnosis is confirmed at laryngoscopy where the haemangioma is visualised as an eccentric mass. Deep biopsy reveals the histology of a capillary haemangioma.

Historically, steroids have been widely used for croup, but support for their role has only recently, been defined. A meta-analysis of over 1100 patients from nine trials supported the use of steroids for unintubated patients. ${ }^{1}$ In 
intubated patients, a prospective study of prednisolone $1 \mathrm{mg} / \mathrm{kg}$ every 12 hours after intubation was shown to reduce the duration of intubation (from 138 hours to 98 hours), lead to fewer doses of nebulised adrenaline required after extubation, and a reduced need for reintubation. ${ }^{2}$ A significant number of patients were extubated within 72 hours of intubation, even in the absence of a leak. The recommendation was that $0.6 \mathrm{mg} / \mathrm{kg}$ dexamethasone should be followed by prednisolone $1.0 \mathrm{mg} / \mathrm{kg}$ every 12 hours for the duration of intubation.

Systemic corticosteroids may also be used for the treatment of haemangiomata: a report from Johns Hopkins Hospital in 1967 first documented their use for non-airway lesions. ${ }^{3}$ Cohen and Wang first reported on the use of systemic corticosteroids for airway lesions in $1977 .{ }^{4}$ Although effective, they may be needed for a prolonged period.
In both of our patients, the initial diagnosis of LTB led to the use of systemic corticosteroids, causing the resolution of symptoms of the airway haemangiomata. Cessation of the steroids resulted in symptomatic deterioration. Formal airway evaluation should be undertaken early in such cases. An airway haemangioma should be considered in every child with a cutaneous haemangioma who presents with croup.

1 Kairys SW, Olmstead EM, O'Conner GT. Steroid treatment in laryngotracheobronchitis: a meta-analysis of the evidence from randomised trials. Pediatrics 1989; 83: 683-93.

2 Tibballs J, Shann FA, Landau LI. Placebo-controlled trial of prednisolone in children intubated for croup. Lancet 1992; 340: 745-8.

3 Zarem HA, Edgerton MT. Induced resolution of cavernous haemangiomas following prednisone therapy. Plast Reconstr Surg 1967; 39: 76-83.

4 Cohen SR, Wang CI. Steroid treatment of haemangiomas of the head and neck. Ann Otol Rhinol Laryngol 1972; 83: 584-90. 